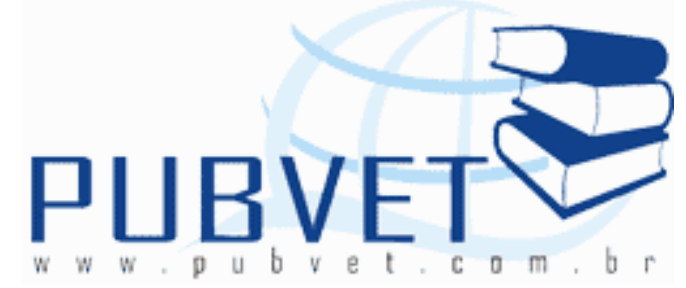

PUBVET, Publicações em Medicina Veterinária e Zootecnia.

Disponível em: <https://doi.org/10.31533/pubvet.v02n11a445>.

\title{
Objetivos e critérios de seleção em bovinos de corte
}

Jorge Luís Ferreira ${ }^{1}$ e Arcadio de los Reyes Borjas ${ }^{2}$

1Universidade Federal do Tocantins, Escola de Medicina Veterinária e Zootecnia, Campus de Araguaína, TO.

2Universidade Federal de Goiás. Escola de Veterinária. Campus II, GO.

\section{INTRODUÇÃO}

A produção de carne de forma eficiente é o principal objetivo das criações domésticas de bovinos de corte. Assim, o estudo detalhado das fases de ganho de peso mostra como as condições genéticas e de manejo podem influenciar a produção desses animais. As pressões exercidas pelo mercado e a necessidade de se produzir carne de boa qualidade, de forma eficiente e competitiva, têm estimulado pecuaristas e pesquisadores a buscarem alternativas de critérios de seleção para precocidade, tanto de crescimento, sexual como de acabamento. No entanto, esses conceitos não podem ser utilizados indistintamente, pois certamente essas características estão muito correlacionadas.

Aumentos na produção e na produtividade da carne e no rendimento industrial de seus produtos visando corrigir o déficit crônico destes, requerem incrementos no efetivo nacional, melhorias rápidas e significativas nos 
Ferreira, J.L. e Borjas, A.R. Objetivos e critérios de seleção em bovinos de corte. PUBVET, Londrina, V. 2, N. 45, Art\#445, Nov2, 2008.

sistemas de criação dos rebanhos e na base genética. Para se avaliar, no âmbito técnico e econômico, os modelos produtivos vigentes e a adoção de novas práticas de produção animal, têm sido reconhecidos como vital determinar economicamente o potencial sustável dos sistemas produtivos (BITTENCOURT et al. 1998). Desse modo, dentre os conhecimentos aplicáveis a elevar a eficiência produtiva dos rebanhos, o melhoramento animal tem adquirido interesse proeminente.

Nos últimos anos, surgiram e se desenvolveram no Brasil diversos programas de melhoramento genético de bovinos de corte. Nestes programas, após a coleta e organização dos dados junto aos participantes, é realizada, em geral, a avaliação genética dos animais, visando fornecer aos criadores participantes informações auxiliares para o processo de seleção. As informações geradas podem variar muito de acordo com o programa, o criador e o mercado. Entretanto nestes programas as avaliações genéticas periódicas foram desenvolvidas sem uma definição "a priori" dos objetivos de seleção.

A maximização do lucro é o objetivo lógico para a maioria dos programas de melhoramento genético. Entretanto há uma questão que deve ser resolvida, para quem o programa de melhoramento animal deve maximizar o lucro? 0 criador, o invernista, a indústria ou o consumidor? Qualquer atividade produtiva tem como objetivo a obtenção do lucro e como tal produtores de rebanhos de corte, para permanecerem no negócio, devem gerir a sua atividade de forma rentável e competitiva. O caminho do êxito de uma pecuária produtiva reside na utilização racional dos recursos genéticos e ambiental de maneira a maximizar o retorno liquido (lucro) (MCMANUS \& THOMPSON,1993). De acordo com HILL (1974) o melhoramento animal consiste basicamente na identificação dos objetivos de seleção, identificação dos indivíduos que produzirão a próxima geração, seleção efetiva na progênie e utilização do sistema de acasalamento adequado. Portanto, a definição dos 
Ferreira, J.L. e Borjas, A.R. Objetivos e critérios de seleção em bovinos de corte. PUBVET, Londrina, V. 2, N. 45, Art\#445, Nov2, 2008.

objetivos de seleção deveria ser o primeiro passo na elaboração de um programa de melhoramento genético.

Apesar do inegável reconhecimento de suas contribuições para dinamizar a pecuária de corte, os poucos programas de melhoramento genético existentes apresentam uma evidente deficiência na caracterização dos objetivos de seleção, ou seja, de determinar os rumos que as práticas de seleção devem permear, no intuito de maximizar o retorno econômico da atividade. Estudos que concatenam essas duas áreas do conhecimento (genética e econômica) são incipientes no Brasil, e a escolha de reprodutores baseia-se essencialmente na avaliação visual, e/ou muito raramente em avaliações genéticas, como exemplo em DEP's (Diferença Esperada na Progênie).

O presente trabalho tem como objetivo discutir sobre objetivos e critérios de seleção em rebanhos bovinos de corte, e sua contribuição para o aprimoramento dos programas de melhoramento genético.

\section{REVISÃO DE LITERATURA}

\subsection{Objetivos de seleção e ponderadores econômicos}

Atualmente, a obtenção de estimações/predições de valores genéticos dos animais para um grande número de características é rotineira, e os futuros reprodutores podem ser obtidos em acasalamentos planejados. Entretanto, cada vez é mais difícil para o criador escolher quais as características a serem utilizadas como critérios de seleção e, qual a importância relativa a ser dada a cada uma delas, já que os sistemas de produção são variáveis e o melhoramento genético deve se feito para um dado ambiente de produção. Como o objetivo de todo sistema produtivo é o de maximizar o lucro, muitas vezes tornar-se difícil definir os objetivos de seleção. 
Ferreira, J.L. e Borjas, A.R. Objetivos e critérios de seleção em bovinos de corte. PUBVET, Londrina, V. 2, N. 45, Art\#445, Nov2, 2008.

Assim, o primeiro passo num programa de melhoramento consiste na derivação formal dos seus objetivos econômicos. Uma vez que são várias as características que afetam a eficiência econômica, e sendo a unidade de seleção, o individuo reprodutor (macho ou fêmea), a seleção artificial implica na ponderação das diferentes características que se deseja melhorar. Em um primeiro momento faz-se necessário, conhecer e estabelecer qual o extrato produtivo que está sendo visado, pois em muitos casos, pode haver conflitos entre os objetivos nos diferentes elos da cadeia produtiva (criador-produtorindustria-consumidor), dificultando ainda mais, a tomada de decisão (MOAV, 1966; PEARSON, 1982).

A definição do objetivo de seleção é o primeiro passo para a resolução deste problema, e o seguinte é a construção de um índice para determinar a lista de características que influenciam o objetivo e determinar a importância relativa de cada característica desta lista (GOLDEN \& BOURDON, 1999).

Toda a base genético-econômica para a seleção de várias características simultaneamente foi desenvolvida por HAZEL (1943) em seu trabalho clássico da Teoria do Índice de Seleção. Nesta teoria, o autor considerou que o valor relativo de cada característica depende da quantidade de aumento no lucro que pode ser esperada para cada unidade de melhoramento naquela característica. Dentro desta estrutura, os fatores de ponderação que representam as características econômicas das mudanças nos critérios de seleção do rebanho são combinados com os fatores de ponderação genéticos representando o quanto as práticas de seleção podem causar melhorias em cada característica. O diagrama de trilha exemplifica bastante as várias relações entre $\mathrm{H}$ e a mensuração fenotípica de cada característica (xi), conforme pode ser observado na Figura 1. 


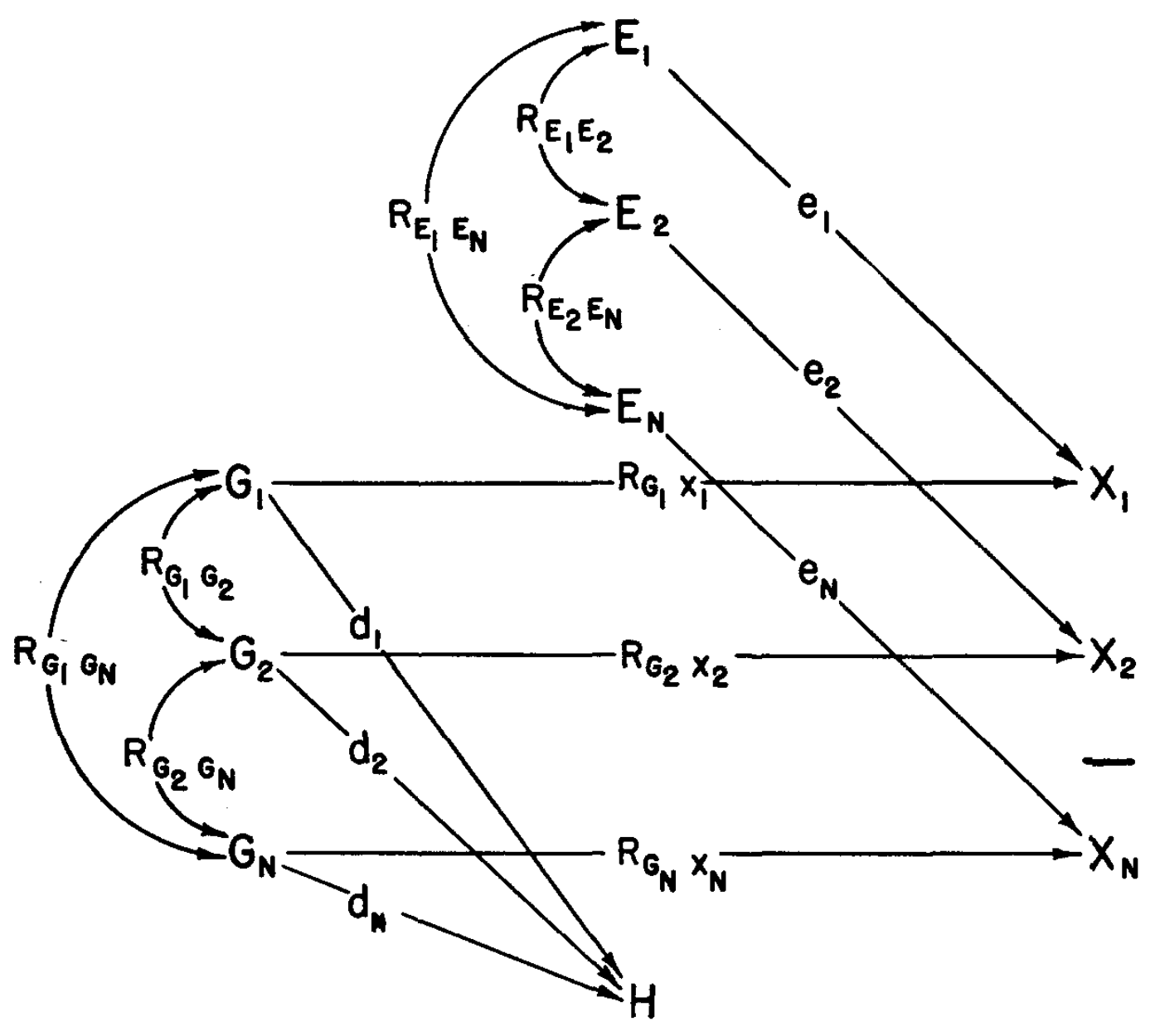

FIGURA 1. Diagrama do coeficiente de trilha mostrando as relações entre mensurações fenotípicas (xi) e genótipo agregado $(H)$.

Fonte: HAZEL (1943).

Pela figura nota-se que os valores genéticos e fenotípicos variam para cada uma das características. Sendo que o valor agregado pode ser obtido pelo somatório dos vários genótipos (assumindo a distinção genotípica de cada característica) existentes correlacionando com o valor econômico da característica. Este genótipo agregado pode ser representado pela fórmula:

$$
H=a_{1} G_{1}+a_{2} G_{2}+\ldots+a_{n} G_{n}
$$

Apesar de o trabalho ser bastante conhecido e a metodologia estar disponível há mais de 60 anos, poucos são os trabalhos enfocados nesta área. 
Ferreira, J.L. e Borjas, A.R. Objetivos e critérios de seleção em bovinos de corte. PUBVET, Londrina, V. 2, N. 45, Art\#445, Nov2, 2008.

WELLER (1994) afirma que um dos principais motivos da não atenção de pesquisadores, produtores e profissionais ligados ao segmento da cadeia produtiva, deve-se aos princípios básicos do índice de seleção, que não permite que se obtenha uma solução completa para a determinação do objetivo de seleção, uma vez, que se assume serem os pesos econômicos relativos de cada característica conhecidos. Neste caso, o índice de seleção tradicional é de pouca utilidade para a determinação dos objetivos de seleção.

HAZEL (1943) definiu "mérito genético agregado" como $H=\Sigma a_{i} G_{i}$, em que $\mathrm{H}$ é uma função linear dos valores genéticos para cada uma das características de interesse $\left(G_{i}\right)$ ponderadas pelo seu valor econômico $a_{i}$. Este autor definiu os valores econômicos $a_{i}$ como "a quantidade esperada de aumento no lucro por cada unidade de melhoramento na característica i". Assim, foi introduzido o conceito de genótipo agregado ou do objetivo de seleção $(H)$, que foi definido como uma função linear dos valores genéticos ponderados pelos valores econômicos de cada característica. Dentro desta estrutura, os fatores de ponderação que representam o objetivo de seleção do rebanho (valores econômicos) são combinados com os fatores de ponderação genéticos, representando o quanto as práticas de seleção podem causar melhorias em cada característica.

Posteriormente, MOAV \& HILL (1966) generalizaram este raciocínio à situação mais comum de não-linearidade do lucro, expresso como função das características zootécnicas, e definiram os valores econômicos de cada característica como a derivada parcial da função de lucro com respeito á característica em questão, avaliada nos valores médios atuais das outras características. O objetivo de seleção é função de características que se deseja melhorar, sendo esta aplicada com base num critério de seleção (JAMES, 1982).

PONZONI (1986) caracteriza o desenvolvimento dos objetivos de seleção, seguindo as fases de:

1. Especificação do sistema produtivo, mercado e reprodutivo; 
Ferreira, J.L. e Borjas, A.R. Objetivos e critérios de seleção em bovinos de corte. PUBVET, Londrina, V. 2, N. 45, Art\#445, Nov2, 2008.

2. Identificação das fontes de rendimentos e despesas no rebanho comercial;

3. Determinação das características biológicas influenciando os rendimentos e despesas e;

4. Estimação de valores econômicos para cada característica componente dos objetivos de seleção.

Segundo HARRIS (1998) posicionar os objetivos de seleção nas formas matemáticas, em uma completa base econômica, é a chave para integrar, modernamente o desenvolvimento da seleção animal dentro de programas industriais. Para que o objetivo de seleção seja estabelecido, deve-se, inicialmente, definir o mercado que se deseja atingir, uma vez que as características a serem selecionadas dependem do produto a ser comercializado e, igualmente, de como os animais são manejados, alimentados, etc. Com isso, o conjunto de informações referentes a custos, receitas e dados de produção, tende a ser o mais completo possível e condizente com a rotina e as particularidades de cada propriedade.

PARNELL (2000) revela que a caracterização dos objetivos de seleção envolve a necessidade de identificação dos níveis atuais de produtividade média dos animais, como forma de estabelecer metas de desempenho futuro para as características a serem selecionadas. Comenta ainda, que o desempenho atual com as metas futuras, de acordo com o mercado, pode estabelecer as características a serem enfatizadas no processo seletivo. Para tanto o produtor deve realizar um estudo da situação atual de seu rebanho e confrontar com o que os consumidores finais desejam. Demonstrando que o conhecimento do nível de produção implica na necessidade de colheita de dados de produção e informações dos compradores a respeito do desempenho de seus animais, o que talvez seja o mais difícil em muitas situações práticas.

Neste sentido PARNEL (2000) sugere as seguintes etapas a serem seguidas:

Etapa 1. Características de importância econômica: onde estas devem atender as exigências do mercado, além de atender as expectativas de 
Ferreira, J.L. e Borjas, A.R. Objetivos e critérios de seleção em bovinos de corte. PUBVET, Londrina, V. 2, N. 45, Art\#445, Nov2, 2008.

melhoria do próprio rebanho. Estando ciente da necessidade de flexibilização de produção.

Etapa 2. Conhecimento da situação real da propriedade, podendo assim otimizar o uso de insumos, técnicas e infra-estrutura necessária para alcançar tais metas. A Caracterização do empreendimento serve de ponto de partida para o direcionamento dos próximos passos e principalmente definição dos objetivos de seleção.

Etapa 3. Determinação dos critérios de seleção que são os meios pelos quais os objetivos serão alcançados.

GOLDEN \& BOURDON (1999) discute a importância de se estimar o valor genético para todas as características economicamente importantes no objetivo de seleção, independentemente se as características fenotípicas são ou não avaliadas, pois características de relevância econômica são caracteres que influem no rendimento da produção.

Um ponto extremamente importante é o fato de que um dos principais obstáculos para a aplicação dos objetivos de seleção tem sido a correta determinação dos valores econômicos das diferentes características, uma vez não estar totalmente claro como estimar os valores econômicos das características que afetam a quantidade de produto e as características que afetam os custos de produção. Vários autores afirmam que a determinação dos ponderadores econômicos é essencial na predição da rentabilidade dos programas de seleção.

O valor econômico (VE) pode ser calculado como a variação de lucro inerente ao respectivo melhoramento no desempenho médio simulado da característica (representando o ganho genético médio da característica), enquanto mantendo todas as demais variáveis componentes do modelo bioeconômico constantes.

Um modelo foi proposto por HARRIS (1970) onde se obtém a derivação formal de valores econômicos. Neste o peso econômico de uma característica é o retorno bruto por unidade mudança. Renda $(R)$ e Despesa $(D)$ podem ser combinadas de maneiras diferentes, para estimar valores econômicos. Neste 
Ferreira, J.L. e Borjas, A.R. Objetivos e critérios de seleção em bovinos de corte. PUBVET, Londrina, V. 2, N. 45, Art\#445, Nov2, 2008.

modelo, Lucro $(L)=R-D(1)$; Retorno no investimento $(\Phi)=R / D(2)$; e Custo por unidade de produção $(Q)=D / R$ (3). Quando renda e despesas são independentes dos custos fixos, eles somam com diferenciação.

JAMES (1982) sugere que ao longo do tempo a eficiência de produção ( $\Phi$ ou Q) é o critério apropriado, enquanto SMITH et al. (1986) coloca que lucro real vem da redução de custos por unidade de produção. Estes mesmos autores concluíram ainda, que $\Phi$ ou $Q$ são mais apropriadas, porque lucro tem um componente que pode ser conseguido com mudança em escala do empreendimento, sem melhoramento genético, mas as diferenças entre os vários métodos podem ter um efeito pequeno em termos práticos.

MORRIS (1980) mostrou que o lucro é o maior incentivo para mudanças no empreendimento da fazenda, já BRASCAMP et al. (1985) e PONZONI (1992) mostraram que, se o lucro é colocado como 0 (zero), os três métodos são iguais e usando $L$ é melhor porque é mais simples. Os valores econômicos são encontrados expressando $L$ como função das características no objetivo de seleção e usando derivação parcial de $L$ em respeito da característica em questão (calculado na média das outras características). Isto tem o efeito de linearizar a equação. Este procedimento é realizado supondo que mudanças genéticas são vagarosas e, portanto, sobre um período curto de tempo, serão essencialmente lineares (JAMES, 1986). Em outros estudos MCMANUS \& THOMPSON (1993) mostraram que as correlações entre os valores usando os três métodos (1, 2 e 3 ) são altas.

Para o modelo de cálculo dos Valores Econômicos, WELLER (1994) propôs uma fórmula para a metodologia de modelo bioeconômico, como segue:

$$
\mathrm{VE}=\Delta \mathrm{L} / \Delta \mathrm{G}
$$

Onde, $\mathrm{VE}=$ valor econômico da característica;

$\Delta \mathrm{L}=$ variação de lucro;

$\Delta \mathrm{G}=$ variação no desempenho produtivo da característica (ganho genético).

Entretanto, nem todas as características no objetivo de seleção são expressas na mesma freqüência ou no mesmo tempo (PONZONI, 1988). Os 
Ferreira, J.L. e Borjas, A.R. Objetivos e critérios de seleção em bovinos de corte. PUBVET, Londrina, V. 2, N. 45, Art\#445, Nov2, 2008.

métodos usados que levam isto em conta incluem: calcular renda e despesa em um ano (levam em consideração freqüência, mas não tempo de resposta) ou usar taxas descontadas de fluxo de genes (JAMES, 1982, MCCLINTOCK \& CUNNIGHAM, 1974, PONZONI \& NEWMAN, 1989, MCMANUS \& THOMPSON, 1993). Este método calcula fluxo de caixa e depois desconta de volta para o presente valor, isto é necessário porque renda volta em tempos diferentes.

O fluxo de genes descontado é expresso como número de expressões cumulativas descontadas (CDE), resultantes de um cruzamento; "cumulativo" referente a uma acumulação das expressões sobre gerações ou anos; e "descontado" implica ao fato que o retorno futuro é descontado ao valor de hoje por um fator de desconto (BRASCAMP, 1978). Como exemplo, CUNNINGHAM \& RYAN (1975), baseado na sua pesquisa em bovinos, concluíram que todos os benefícios de uma inseminação são completos dentro de 20 anos, com aproximadamente $90 \%$ sendo realizado nos primeiros dez anos, quando se usa a taxa de $10 \%$. Provavelmente, devido a isso, um horizonte de 10 a 20 anos foi escolhido em estudos, como de DANELL et al. (1976), WILTON \& DANELL (1981) e GROEN (1990).

Um outro modelo foi proposto por BRASCAMP et al. (1985), utilizando o método de Equações de Lucro, que pode ser visualizado, conforme modelo:

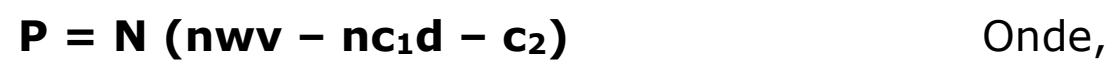

$P=$ lucro;

$\mathrm{N}$ = número de matrizes em reprodução;

$\mathrm{N}$ = número de progênie por matriz ao ano;

$\mathrm{w}=$ peso da progênie;

$\mathrm{v}=$ valor por unidade da característica considerada;

$\mathrm{C}_{1}=$ custo ao dia de crescimento da progênie;

$\mathrm{C}_{2}=$ custo por matriz ao ano;

$\mathrm{d}=$ idade ao peso considerado.

Para o modelo acima, as características cujo valor econômico podem ser estimadas, são: número de progênies por matriz ao ano (n), peso da progênie (w) e idade ao peso considerado (d). Os valores econômicos, para esse 
Ferreira, J.L. e Borjas, A.R. Objetivos e critérios de seleção em bovinos de corte. PUBVET, Londrina, V. 2, N. 45, Art\#445, Nov2, 2008.

método, são obtidos através da derivação parcial de equações de lucro com respeito a cada característica de influência genética, como apresentado na Tabela 1.

Tabela 1. Equação de lucro e respectiva derivada, para as características avaliadas.

Equações de Lucro Valores econômicos

$\begin{array}{llll} & n^{1} & n^{2} & n^{3} \\ =N\left(n w v-n c_{1} d-c_{2}\right) & N\left(w v-c_{1} d\right) & -N n c_{1} & N n v\end{array}$

1. derivada do lucro para o número de progênies por matriz ao ano.

2. derivada do lucro para peso da progênie.

3. derivada do lucro para idade ao peso considerado.

Fonte: Adaptado de GIBSON \& VAN ARENDONK (1998).

O primeiro passo para a estimativa de ponderadores econômicos é a definição de cada parâmetro genético, as receitas e custos do sistema de produção em um período fixo de tempo (BITTENCOURT et al. 1998). Vários autores apontam que a estimação do lucro pode ser bastante complexa sob certas circunstâncias e depende das características sob seleção e da força do mercado.

\subsection{Características de objetivos e critérios de seleção}

PARNEL (2000) revela que a caracterização dos objetivos de seleção envolve a necessidade dos níveis atuais de produtividade média dos animais, como forma de estabelecer metas de desempenho futuro para as características a serem selecionadas. Neste caso, quando compara-se o desempenho atual com as metas futuras, de acordo com o mercado, pode-se identificar as características a serem enfatizadas na seleção.

Muitas características avaliadas nos animais (critérios de seleção) não afetam diretamente o lucro, sendo aplicadas para indicar o mérito genético do animal para outras características. As características denominadas indicadoras 
Ferreira, J.L. e Borjas, A.R. Objetivos e critérios de seleção em bovinos de corte. PUBVET, Londrina, V. 2, N. 45, Art\#445, Nov2, 2008.

são portanto, recursos para se tentar melhorar características do objetivo do produtor.

PHOCAS et al. (1998) alertam ao fato que podem ocorrer diferenças entre os valores econômicos por se trabalhar com distintas metodologias, circunstâncias de criação e produção, além das características avaliadas. Com isso torna-se difícil a comparação entre os diversos estudos realizados, uma vez que além dos fatos citados, os sistemas de produção variam conforme a região estudada. Quando de compara estudos que envolveram características de reprodução, crescimento (produção) e carcaça se verifica distinções entre estes, conforme pode ser observado na Tabela 2.

Tabela 2. Comparação entre as proporções econômicas encontradas para as características de reprodução, crescimento e carcaça.

\begin{tabular}{|c|c|c|c|}
\hline Autores & $\begin{array}{l}\text { Característica } \\
\text { s reprodutivas }\end{array}$ & $\begin{array}{l}\text { Característica } \\
\text { s crescimento }\end{array}$ & $\begin{array}{c}\text { Característica } \\
\text { s carcaça }\end{array}$ \\
\hline $\begin{array}{lll}\text { BARWICK } & \text { et } & \text { al. } \\
(1994) & & \end{array}$ & 3 & 2 & 1 \\
\hline NITTER et al. (1994) & 6 & 2 & 1 \\
\hline $\begin{array}{l}\text { MACNEIL et al. } \\
(1994)\end{array}$ & 4 & 1 & 1 \\
\hline $\begin{array}{l}\text { NEWMAN et al. } \\
(1994)\end{array}$ & 2 & 1 & - \\
\hline
\end{tabular}

PHOCAS et al. (1998) observaram uma relação de 1,5 vezes mais importante para as características de fertilidade em relação às características de produção. Já BITTENCOURT et al. (2006), chegou a um valor para características de fertilidade de 3,86 vezes mais importante economicamente que peso a desmama. FORMIGONI et al. (2005) observou que dentre as características de fertilidade avaliadas, estas foram 1,19 a 1,71 vezes mais importantes economicamente que a característica de peso a desmama, sendo 
Ferreira, J.L. e Borjas, A.R. Objetivos e critérios de seleção em bovinos de corte. PUBVET, Londrina, V. 2, N. 45, Art\#445, Nov2, 2008.

que os valores genético-econômicos encontrados foram de 10,82 para habilidade de permanência de vacas, 7,73 para probabilidade de prenhes em novilhas aos 14 meses e 10,82 para peso a desmama. Diversos autores Van der WERF \& KINHORN (2001); AMER et al. (1998) e PHOCAS et al (1998) demonstraram valores econômicos positivos para características de conformação de carcaça e taxas de crescimento.

AMER et al. (2001) em estudos sobre objetivos de seleção em bovinos de corte na Irlanda, mostraram variações substanciais nos VE de características de fertilidade, características de carcaça e de reprodução. Diversos estudos relacionando estas características em bovinos de corte têm sido descritos na literatura (PORTOLANO et al. (2006); HANFORD et al. (2006)).

Segundo QUEIROZ et al. (2005) um outro ponto a ser considerado é que, muitas vezes, a característica que pode ser medida no animal tem pouco impacto no objetivo econômico da seleção, ou então, as características economicamente relevantes podem ter várias características indicadoras. A escolha de critérios de seleção corretos e a maneira como os mesmos devem ser ponderados parecem ser as decisões mais importantes a ser tomadas pelo produtor.

Nos Estados Unidos até recentemente tinha-se considerado em programas de melhoramento genético de ovinos de corte apenas o custo de alimentação e a taxa de crescimento como economicamente importantes (SNOWDER \& VAN VLECK, 2003). Estes autores demonstraram que a seleção para eficiência alimentar mostrou superioridade em relação à seleção para consumo alimentar, denotando que esta característica tem maior peso econômico, e que deve ser inserida em índices de seleção. A eficiência de um índice é mensurada pela correlação entre o índice e os objetivos de seleção. As características são adicionadas ou removidas de um índice, dependendo das mudanças dos valores das correlações. Essas mudanças determinam com que combinações as características se apresentam e podem ser usadas para a tomada de decisões. 
Ferreira, J.L. e Borjas, A.R. Objetivos e critérios de seleção em bovinos de corte. PUBVET, Londrina, V. 2, N. 45, Art\#445, Nov2, 2008.

Assim, o critério de seleção será naturalmente influenciado pelas características do objetivo de produção, sendo o inverso uma prática condenada, uma vez que isso poderia levar a omissão de características economicamente importantes. A identificação das características a serem selecionadas, assim como a sua importância econômica para um dado sistema de produção, pode ser mais bem ilustrada na Tabela 3, conforme exemplificado por PARNELL (2000).

Tabela 3. Exemplo do estabelecimento de objetivos e respectivos critérios de seleção, para o sistema de cria, segundo PARNELL (2000).

\begin{tabular}{lcc}
\hline Características & Objetivos de seleção & Critérios de seleção \\
\hline Fertilidade & aumento da fertilidade & $\begin{array}{c}\text { Precocidade sexual novilhas } \\
\text { Habilidade de permanência }\end{array}$ \\
\hline Peso a desmama & aumento peso de venda & Peso a desmama \\
\hline
\end{tabular}

Fonte: adaptado de PARNELL (2000).

Em bovinos, as características de importância econômica podem ser divididas em duas categorias: primeira, as que afetam diretamente o lucro, como dias para o abate, peso corporal, entre outras, e a segunda que apresenta influencia indireta e subjetiva no lucro, como exemplo aquelas de difícil mensuração. A Tabela 4 lista uma séria de características de relevância econômica direta e indireta, segundo BITTENCOURT (2001).

FORMIGONI et al. (2005) em estudo sobre valores econômicos para características inclusas em critérios de seleção de gado de corte, observaram que a característica habilidade de permanência no rebanho apresentou superioridade econômica em termos absolutos quando comparada a característica de probabilidade de prenhez aos 14 meses. Revelando ainda, que os fatores de produção que interferem no valor econômico da habilidade de permanência (HP) devem ser igualmente avaliados por meio de modelos bioeconômicos. 
Ferreira, J.L. e Borjas, A.R. Objetivos e critérios de seleção em bovinos de corte. PUBVET, Londrina, V. 2, N. 45, Art\#445, Nov2, 2008.

Tabela 4. Lista de características indicadoras de relevância econômica, segundo BITTENCOURT (2001).

\begin{tabular}{|c|c|c|}
\hline Características & & Indicadores $^{1}$ \\
\hline \multicolumn{3}{|l|}{ Peso animal } \\
\hline & A desmama (materno) & Peso aos 120 dias \\
\hline & A desmama (direto) & Peso aos 210 dias \\
\hline & \multirow[t]{4}{*}{ Ao abate } & Peso aos 365 dias \\
\hline & & Peso aos 450 dias \\
\hline & & Peso aos 550 dias \\
\hline & & Peso da carcaça \\
\hline \multicolumn{3}{|c|}{ Peso da carcaça } \\
\hline & & $\begin{array}{l}\text { Peso aos } 450 \text { dias } \\
\text { Peso aos } 550 \text { dias } \\
\text { Cobertura de gordura } \\
\text { Peso da vaca ao descarte }\end{array}$ \\
\hline \multicolumn{3}{|c|}{ Probabilidade de ocorrência de partos normais } \\
\hline & & $\begin{array}{l}\text { Escore para facilidade de parto } \\
\text { Peso ao nascer } \\
\text { Período de gestação }\end{array}$ \\
\hline \multicolumn{3}{|c|}{ Requerimento nutricional de mantença (vacas) } \\
\hline & & $\begin{array}{l}\text { Peso adulto da vaca } \\
\text { Escore de condição corporal } \\
\text { Produção de leite } \\
\text { Peso das vísceras }\end{array}$ \\
\hline \multicolumn{3}{|c|}{ Tempo de permanência no rebanho } \\
\hline & & $\begin{array}{l}\text { Data do parto } \\
\text { Dias para o parto } \\
\text { Intervalo de partos } \\
\text { Produção de leite } \\
\text { Habilidade de permanência }\end{array}$ \\
\hline
\end{tabular}

${ }^{1} \mathrm{~A}$ tabela contém exemplos de características que podem ser usadas como indicadores.

Fonte: adaptado de BITTENCOURT (2001). 
Ferreira, J.L. e Borjas, A.R. Objetivos e critérios de seleção em bovinos de corte. PUBVET, Londrina, V. 2, N. 45, Art\#445, Nov2, 2008.

\section{3. Índices de seleção}

HAZEL (1943) desenvolveu a teoria econômica dos índices de seleção (TEIS). Na TIES, o autor considerou que o valor econômico relativo de cada característica depende da quantidade de aumento no lucro que pode ser esperada para cada unidade de melhoramento naquela característica. Dentro desta estrutura, os fatores de ponderação que representam o desejo econômico das mudanças nos critérios de seleção do rebanho são combinados com os fatores de ponderação genéticos, representando o quanto as práticas de seleção podem causar melhorias em cada característica.

Um índice econômico de seleção pode ser entendido como a combinação de fatores de ponderação e informações genéticas, no caso clássico, de valores fenotípicos, de mais de uma característica. Os índices são usados na seleção de características múltiplas para a predição do valor genético agregado. As características inclusas no índice, características indicadoras ou critérios de seleção, devem ser aquelas que podem ser mensuradas com facilidade, baixo custo e mais cedo na vida dos animais, além de apresentarem estimativas de herdabilidade de mediana a alta, contribuírem e estarem relacionadas ao objetivo de seleção. Os índices econômicos de seleção usando valores fenotípicos não são capazes de levar em conta diferenças genéticas entre rebanhos ou grupos contemporâneos (GC). Portanto, devem ser utilizados somente quando as informações fenotípicas forem provenientes de GC geneticamente similares. Salienta-se que as informações fenotípicas usadas para a construção desses índices devem ser limitadas a um período de tempo relativamente curto, para evitar que a mudança genética não cause diferença acentuada entre os GC.

A seleção de múltiplas características adotando-se índices econômicos de seleção é, segundo BOURDON (1998), a maneira mais rápida e eficiente de melhorar o valor genético agregado, porque utiliza uma grande quantidade de informação de várias características para produzir um valor único - o valor do 
Ferreira, J.L. e Borjas, A.R. Objetivos e critérios de seleção em bovinos de corte. PUBVET, Londrina, V. 2, N. 45, Art\#445, Nov2, 2008.

índice que prediz o mérito genético econômico médio de um indivíduo. Ele torna fácil a classificação dos indivíduos, porém não caracteriza o animal em detalhes. O índice tenta conciliar a seleção para características que possuem relações genéticas antagônicas, mas o resultado nem sempre favorece o ganho genético para todas as características. O emprego de índices evidencia a direção em que a seleção está sendo processada, mas não o alvo a ser atingido. Ocasionalmente, a utilização de índices de seleção pode resultar na seleção de indivíduos extremos, que seriam desaconselháveis para o melhoramento em uma ou mais características (por exemplo, peso ao nascer). Nesta situação, segundo BOURDON (1998), poder-se-ia associar a utilização do índice com outro método de seleção de múltiplas características, como o dos níveis independentes de rejeição, para eliminar estes indivíduos extremos.

QUEIROZ et al. (2005) trabalhando com dois índices de seleção para um rebanho Caracu de duplo propósito, verificaram que os dois índices econômicos de seleção trariam considerável resposta para o objetivo proposto. Entretanto o índice 1 , que incluiu peso a desmama, seria um pouco mais eficiente em termos de resposta à seleção total. Os autores ainda salientam que as informações fenotípicas usadas para a construção de índices de seleção para animais de duplo propósito, devem ser limitadas a um período curto de tempo, para evitar que a mudança genética não cause diferença acentuada entre os grupos de contemporâneos.

\section{CONSIDERAÇÕES FINAIS}

Uma das maiores dificuldades para se definir critérios de seleção para bovinos de corte no Brasil é a variedade de sistemas de produção existentes. No Brasil existem diferenças climáticas, topográficas e de qualidade do solo e desigualdades sociais muito grandes, o que propicia uma gama de sistemas de produção. Entretanto, está acontecendo mudanças na direção de maior 
Ferreira, J.L. e Borjas, A.R. Objetivos e critérios de seleção em bovinos de corte. PUBVET, Londrina, V. 2, N. 45, Art\#445, Nov2, 2008.

intensificação dos sistemas de produção, o que deverá facilitar a definição dos objetivos de seleção.

A importância de avaliar as relações de custos e receitas do melhoramento genético do rebanho é de fundamental importância nos processos de tomada de decisão na pecuária de corte nos dias de hoje.

Desconhecer as relações de preços e o impacto econômico da seleção impossibilita o produtor, em projetar o sentido da seleção ou mesmo redirecionar esforços quando necessário, na busca de melhores resultados econômicos com a atividade pecuária de corte. Para tanto, recomenda-se desenvolver e/ou aplicar metodologias de análise de lucro esperado a partir da seleção de acordo com as particularidades de cada sistema produtivo.

\section{REFERENCIAS BIBLIOGRÁFICAS}

1. AMER, P.R. Estimation of economic weights in genetics improvement using neoclassical production theory: an alternative to rescaling. Animal Production, v.54, p.341-50, 1994.

2. AMER, P.R.; SIMM, G.; KEANE, M.G.; DISKIN, M.G.; WICKHAM, B.W. Breeding objectives for beef cattle in Ireland. Livestock production Science, v.67, p.223-39, 2001.

3. BARWICK, S.A.; B-OBJECT: a PC-Program to derive economic weights for beef cattle. In: GENETIC PREDICTION WORSHOP, 4, 1994, Kansas City. Proceedings... Kansas City: Beef Improvement Federation, 1994. 10p.

4. BITTENCOURT, T.C.C. Estimativa de ponderadores econômicos para características de importância econômica em gado de corte, usando equações de lucro. Ribeirão Preto, 2001. 59p. Tese (doutorado) - Faculdade de Medicina de Ribeirão Preto, Universidade de São Paulo, Ribeirão Preto.

5. BITTENCOURT, T.C.C.; LÔBO, R.B.; BEZERRA, L.A.F. Objetivos de seleção para sistemas de produção de gado de corte em pasto: ponderadores econômicos. Arq. Brás. Méd.Vet.Zootec., v.58, n.2,p.196-204, 2006.

6. BITTENCOURT, T.C.C.; LÔBO, R.B.; FIGUEIREDO, L.F.; SILVA, A.C.V. Derivação de ponderadores econômicos para características produtivas em gado de corte usando equações de lucro. In: REUNIÃO ANUAL DA SOCIEDADE BRASILEIRA DE ZOOTECNIA, 35, 1998, Botucatu. Anais... Botucatu: SBZ, 1998. p.422-23.

7. BOURDON, R.M. Shortcomings of current genetic evaluation systems. J. Anim. Sci., v.76, p.2308-2323, 1998.

8. BRASCAMP, E.W. Methods on economic optimization of animal breeding plants. Report B-134, Research Institute for Animal Husbandry "Schoonoord", Zeist, The Netherlands. 1978.

9. BRASCAMP, E.W.; SMITH,C.; GUY, D.R. Derivation of economic weights from profit equations. Animal Production, v.40, p. 175-180, 1985.

10. CUNNINGHAM, E.P., RYAN, J. A note on the effect of discount rate and length of the accounting period on the economic value of genetic improvement in cattle populations.

Animal Production, v.21, p.77-80. 1975. 
Ferreira, J.L. e Borjas, A.R. Objetivos e critérios de seleção em bovinos de corte. PUBVET, Londrina, V. 2, N. 45, Art\#445, Nov2, 2008.

11. DANELL, Ö.E., RÖNNINGEN, K., STRÖM, H., ANDERSSON, K., SUNDGREN, P.E. An extension of the discounted gene flow method with example in pig breeding. Acta Agric. Scand. 26:203-210. 1976.

12. FORMIGONI, I.B.; FERRAZ, J.B.S.; SILVA, J.A.II.V.; ELER, J.P.; BRUMATTI, R.C. Valores econômicos para habilidade de permanência e probabilidade de prenhez aos 14 meses em bovinos de corte. Arq. Brás. Méd.Vet. Zootec., v.57, supl.2, p.220-226, 2005.

13. GIBSON, J.B.; VAN ARENDONK, J. Defining Multiple-Trait Objectives for Sustainable Genetic Improvement. Journal of Animal Science, v.76, p.2303-2307, 1998.

14. GOLDEN, B.L.; BOURDON, R.M. The sire summary of the future. Revista Brasileira de Reprodução Animal, v.23, p.78-81, 1999.

15. GROEN, A.F. Influences of production circumstances on the economic revenue of cattle breeding programs. Animal Production v.51, p.469-480. 1990.

16. HANFORD, K.J.; VAN VLECK, L.D.; SNOWDER, G.D. Estimative of genetic parameters and genetic trend for reproduction, weight, and wool characateristics of Polypay sheep. Livestock Science, 2006, no prelo.

17. HARRIS, D.L. Breeding for efficiency in livestock production: defining the economic objectives. Journal of Animal Science, v.30, p.860-865, 1970.

18. HARRIS, D.L. Livestock improvement: art, science, or industry? Journal of Animal Science, v.76, p. 2294-2302, 1998.

19. HAZEL, L.N. The genetic basis for constructing selection indexes. Genetics v.28, p.476-490, 1943.

20. HILL, W.G. Prediction and evaluation of response to selection with overlapping generations. Animal Production v.18, p.117-139. 1974.

21. JAMES, J.W. Economic aspects of developing breeding objectives: general considerations. In: Future developments in the genetic improvement of animals. Ed. JSF Barker, K. Hammond, AE McClintock. P.107-118, Academic Press, Sydney, 1982.

22. JAMES, J.W. Economic evaluation of breeding objectives in sheep and goats - general considerations. $3^{\text {rd }}$ World Congress on Genetic Applied to Livestock Production. Proceedings....Lincoln, Nebraska, v.IX, p.470-478, 1986.

23. MacNEIL, M.D.; NEWMAN, S.; ENNS, R.M.; STEWART-SMITH, J. Relative economic values for Canadian beef production using specialized sire and lines. Can. J. Anim. Sci., v.74, p. 411-17, 1994.

24. MCCLINTOCK, A.E.; CUNNINGHAM, E.P. Selection in dual purpose cattle populations: defining the breeding objective. Animal Production v.18, p.237-247, 1974.

25. MCMANUS, C., THOMPSON, R. Breeding objectives for red deer. Animal Production, v.57, p.161-167, 1993.

26. MOAV, R.V.; HILL, W.G. Specialized sire and dam lines. IV. Selection within lines. Animal Production, v.8, p.375-90, 1966.

27. MORRIS, C. A. Some benefits and costs of genetic improvement in New Zealand sheep and beef cattle industry. N. Zeal. Exp. Agric., v.8, p.331-340, 1980.

28. NEWMAN, S.; MACNEIL,M.; GOLDEN, B.L.; BARWICK, S.A. Implementation and use of selection indexes in genetic evaluation schemes for beef cattle. In: GENETIC PREDICTION WORSHOP, 4, 1994. Kansas City. Proceedings...Kansas City: Beef Improvement Federation, 1994, 11p.

29. NITTER, G.; GRASER, H.U.; BARWICK, S.A. Cost-benefit analysis of increased intensity of recording in the Australian national beef recording scheme. In: WORLD CONGRESS OF GENETICS APPLIED TO LIVESTOCK PRODUCTION, 5. Proceedings..., Sidney, Austrália, 1994. p. 205-8, 1994.

30. PARNELL, P. Balancing growth, carcase and fertility in your breeding program. In: THE ANNUAL FEEDER STEER SCHOOL, 5,2000, Armidale. Proceedings...Armidale: Australian Angus, 2000. p.90-6.

31. PEARSON, R.E. Econonomic aspects of the choice of breeding objective. $2^{\text {nd }}$ World Congress on Genetic Applied to Livestock Production. Proceedings..., v.4, p.50-59, 1982. 
Ferreira, J.L. e Borjas, A.R. Objetivos e critérios de seleção em bovinos de corte. PUBVET, Londrina, V. 2, N. 45, Art\#445, Nov2, 2008.

32. PHOCAS, F.; BLOCH, C.; CHAPELLE, P.; BÉCHEREL, F.; RENAND, G.; MENISSIER, F. Developing a breeding objective for a French purebred beef cattle selection programme. Livestock Production Science, v.57, p.49-65, 1998.

33. PONZONI, R.W. A profit equation for the definition of breeding objective of Australian Merino Sheep. Journal of Animal Breeding and Genetics v.103, p.342-357, 1986.

34. PONZONI, R.W. Genetic Improvement of Hair Sheep in the Tropics. FAO Anim. Prod. And HIth. Paper, 101, Rome, Italy, 1992.

35. PONZONI, R.W. The derivation of economic values combining income and expense in different ways: an example with Australian Merino sheep. Journal of Animal Breeding and Genetics v.105, p.143-153, 1988.

36. PONZONI, R.W., NEWMAN, S. Developing breeding objectives for Australian beef cattle production. Animal Production, v.49, p.35-47, 1989.

37. PORTOLANO, B.; FINOCCHIARO, R.; van KAMM, J.B.C.H.M. Comparason of selection criteria for milk yield traits of Valle del Belice dairy sheep. Livestock Science, v.99, p.277-84, 2006.

38. QUEIROZ, S.A.; PELICIONI, L.C.; SILVA, B.F.; SESANA, J.C.; MARTINS, M.I.E.G.; SANCHES, A. Índices de seleção para um rebanho Caracu de duplo propósito. Rev. Bras. Zootec., v.34, n.3, p. 827-37, 2005.

39. SMITH, C., JAMES, J.W., BRASCAMP, E.W. On derivation of economic weights in livestock improvement. Animal Production v.43, p.545-551. 1986.

40. SNOWDER, G.D.; VAN VLECK, L.D. Estimates of genetic parameters and selection strategies to improve the economic efficiency of postweaning growth in lambs. J. Anim. Sci., v.81, p. 2704-13, 2003.

41. Van der WERF, J., KINGHORN, B., Quantitative genetics. Course notes. Animal Science Department, School of Rural Science and Natural Resources, The University of New England, Armidale, NSW, Australia. 2001.

42. WELLER, J.I. Economics aspects of animal breeding. Chapman \& Hall, 1994.

43. WILTON, J.W., DANELL, O.E. Relative rates of discounted expressions of traits in beef crossbreeding programs. Acta Agricultarae Scandanavia v.31, p.207-216. 1981. 